\title{
Berichterstattung über die Sitzungen der Arbeitsgruppe „Wirtschaftlichkeit und Ethik bei der Osteosynthese“ in der Periode Mai 1999 bis Mai 2000
}

\author{
S. Grafe
}

- Gerechte Mittelverteilung im Gesundheitswesen ist nicht ohne die Neugestaltung und Festigung des Arzt-Patienten-Verhältnisses möglich.

- Die Vertretung des Patienten zur Durchsetzung seiner Rechte ist eine Pflicht des Arztes.

- Der ethische Rahmen ärztlichen Handelns sollte durch die Ärzte selbst gesteckt werden.

Die aktuelle Situation einer angespannten wirtschaftlichen Lage lässt auch die ethischen Fragen in der Medizin und ihre Antworten nicht mehr unberührt. Darüber hinaus hat die Öffentlichkeit sich in den letzten Jahren mehr und mehr tragend, heutzutage auch schon mitbestimmend, in die Diskussion um medizin-ethische Fragen eingeschaltet. Sie fühlt sich, zuständig oder nichtzuständig, auch zu dieser Position im Auftrag des Patienten berufen. Von Seiten der Kosten- und Rechtsträger insonderheit von der Deutschen Krankenhausgesellschaft wird der Ruf immer lauter, Ethikkomitees als Institutionen eines jeden Krankenhauses zu bestellen. Ihr Sinn ist aber weitgehend noch unbekannt und umstritten. Trotzdem wird der Ruf nach einer solchen Institution immer lauter. Die Gefahr der missbräuchlichen Bestimmung ist nicht zu unterschätzen. Wir Ärzte haben nicht mehr viel Zeit, das Heft in die Hand zu nehmen und die Prämissen unseres Handelns von fach- und sachkundiger Position aus ethisch wirksam zu vertreten. Die Arbeitsgruppe der DAO „Wirtschaftlichkeit und Ethik bei der Osteosynthese“ hat sich trotz eines altersbedingten Schwundes deshalb zu einem neuen Aufbruch im neuen Jahrhundert entschieden und neue Mitarbeiter gefunden.

In der letzten Periode wurde es wieder in drei Sitzungen möglich, ein sehr schwieriges Problem anzugehen. Es war aber

OP-JOURNAL 2000; 16: 306

(C) Georg Thieme Verlag Stuttgart · New York klar, dass am Ende keine veröffentlichungsreife Aussage oder ein Statement der AO resultieren konnte und die Diskussion hat gezeigt, dass einer der Kernpunkte der „Mittelverteilung im Gesundheitswesen " in der Bewältigung des eingetretenen Wandels des Arzt-PatientenVerhältnisses liegt. Grundlage für die Diskussion waren zwei Veröffentlichungen. 1. Ein Brief an alle leitenden Krankenhausärzte des Präsidenten des Verbandes der leitenden Krankenhausärzte Deutschlands e.V. vom Juni 1999 und

2. Das Manifest für eine faire Mittelverteilung im Gesundheitswesen vom 13.1.99, erstellt von einer unabhängigen interdisziplinären Arbeitsgruppe in Zürich.

Die Freiheit der Entscheidung zum ärztlichen Handeln am Patienten wird heutzutage durch viele vordergründig als positiv angesehene Verhaltensweisen von uns Ärzten selbst in Frage gestellt. Wenn wir Restriktionen seitens der Wirtschaft, der Rechtsprechung und vor allen Dingen der Öffentlichkeit erfahren müssen und gegen sie uns zur Wehr setzen wollen, müssen wir deshalb unsere eigenen Verhaltensweisen auf den Prüfstand stellen. Wenn Ärzte nur noch nach Gewinnmaximierung streben, stehen Ansehen und Vertrauen auf dem Spiel. Die zum Ende des vergangenen Jahrhunderts mehr und mehr aufgeworfene Frage, welche Rechte hat der Patient, wird leider nicht von den geborenen Anwälten der Patienten, den Ärzten gestellt, sondern von den Medien, so dass sich die Politiker in dieser Frage für kompetent erklärt haben. Dies kommt zum Ausdruck in der Tatsache, dass ein Entwurf einer Charta der Patientenrechte, der im vergangenen Jahr veröffentlicht wurde, die Deutsche Ärztekammer gezwungen hat, nachträglich den Versuch zu unternehmen, die originären Patientenrechte aus ärztlicher Sicht festzuschreiben. Wie in vielen Fällen haben auch hier die Ärzte nicht agiert, sondern nur reagiert, was erfahrungsgemäß einer Schwäche gleich kommt. Eine Sammlung kritischer Fragen an uns selbst, vom Vor- sitzenden, anlässlich der Jahrhundertwende in der ersten Sitzung des neuen Jahres zur Diskussion gestellt, hat uns deshalb auf den Weg gebracht, die Entwicklung der medizinischen Wissenschaften ganz besonders auf dem Gebiet der Osteosynthesen und des Gelenkersatzes im Hinblick auf die Selbstbestimmung ärztlichen Handelns und auf die eigene Entscheidung des Patienten hin zu überprüfen. Als Ergebnis wurde einhellig der obengenannte Kernpunkt, das ArztPatienten-Verhältnis zu beleuchten und aus ihm heraus die vielen Fragen zu beantworten, erkannt. Es wird Zeit, dem Verlust von Kompetenz durch Rückbesinnung auf dieses Verhältnis entgegenzusteuern.

Für die kommende Sitzungsperiode hat sich deshalb die Arbeitsgruppe folgenden patientenorientierten Fragenkomplexen zugewandt:

1. Was bedeutet das Recht auf freie Arztwahl bei der Notsituation nach einem Unfall?

2. Gibt es bei der Osteosynthese für die Therapiewahl durch den Patienten auch Alternativen oder bestimmt das „Internet“" unser Vorgehen?

3. Schafft eine Änderung des Arzthaftungsrechtes bei unfallbedingten und behandlungsabhängigen Misserfolgen bessere finanzielle Hilfen und Unterstützung der Verletzten?

Die Arbeitsgruppe hofft, Antworten zu finden, die speziell aus Sicht des Fachgebietes Traumatologie einen Beitrag für die immer lauter werdende Diskussion um die Patientenrechte, die heute leider nur im Sinne des Verbraucherschutzes gegen den Arzt formuliert wurden, leisten können.

Prof. Dr. med. S. Grafe

Leitender Chefarzt

Ev. Diakonissenkrankenhaus Leipzig gGmbH

Georg-Schwarz-Str. 49

04177 Leipzig 